\title{
BioBlu App: Tecnologia Assistiva para auxiliar o ensino de Genética Clássica a deficientes visuais
}

\author{
Mônica S. de Oliveira ${ }^{1}$, Geovani da S. do Amaral ${ }^{2}$, Juliana Cristina Scheneider ${ }^{3}$, \\ Bruno Merlin'1, Heleno Fülber ${ }^{1}$, Adonney Allan de Oliveira Veras ${ }^{1}$
}

${ }^{1}$ Programa de Pós-Graduação em Computação Aplicada - Universidade Federal do Pará (UFPA) - Tucuruí - PA - Brazil

${ }^{2}$ Faculdade de Engenharia da Computação - Universidade Federal do Pará (UFPA) Tucuruí - PA - Brazil

${ }^{3}$ Programa de Pós-Graduação em Eng. de Barragem e Gestão Ambiental - Universidade Federal do Pará (UFPA) - Tucuruí - PA - Brazil

\{monicasilva@ufpa.br, geovani.af04@gmail.com, julianascheneider@outlook. com, brunomerlin@ufpa.br.com, fulber@ufpa.br, allanveras@ufpa.br

\begin{abstract}
A person with visual impairment faces several challenges throughout his life. One of these can be observed in the school context, where the resource of illustrations can be used to teach some common subjects, for example, in genetics. Thus, this article presents a tool to assist the teaching and learning process of genetics for people with visual impairment. The application was built with the participation of the discipline's teacher and a visually impaired student. The results show a good acceptance by users, being an alternative method of supporting student learning, contributing to their quality of life.
\end{abstract}

Resumo. A pessoa com deficiência visual enfrenta vários desafios ao longo de sua vida. Um destes, pode ser observado no contexto escolar, onde o uso recorrente de ilustrações para o ensino de algumas disciplinas é comum, por exemplo na Genética. Assim, este artigo apresenta uma ferramenta para auxiliar o processo de ensino e aprendizagem da Genética para pessoas com deficiência visual. A aplicação foi construída com a participação de docente da disciplina e aluno com deficiência visual. Os resultados evidenciam uma boa aceitação pelos usuários, sendo um método alternativo de apoio a aprendizagem do aluno, contribuindo para sua qualidade de vida.

\section{Introdução}

A visão humana desempenha uma função vital na vida de todos os indivíduos, uma vez que une a informação sensorial e motora gerada pelo cérebro e corpo, promovendo assim significado e movimento direto, sendo que qualquer alteração neste órgão compromete um sentido fundamental do nosso dia-a-dia [Mendonça et. al, 2008]. Quando estas alterações comprometem o sistema visual ou uma ou mais de suas funções visuais, de forma leve, moderada ou severa, caracteriza-se então a deficiência visual [WHO, 2019].

O deficiente visual enfrenta inúmeras barreiras ao longo de sua vida. Uma destas, acontece durante o início de sua vida escolar. É fato que a inserção de alunos com deficiência visual no contexto regular de ensino é uma realidade desde de 1990, fato que cresce a cada ano [Laplane e Batista, 2008]. Apesar disso, ainda existem grandes desafios 
não só para os educandos, como para os educadores, envolvidos nesse processo inclusivo [Carvalho, 2005], [Fávero e Mantoan, 2007].

Algumas disciplinas, como o ensino das Ciências Biológicas necessita de um enfoque na adoção de materiais e/ou métodos alternativos para a pessoa com deficiência visual, uma vez que ainda observa-se o uso sistemático de ilustrações como estratégia, o que dificulta a aprendizagem de deficientes visuais inseridos em salas de aulas regulares [Cardinali e Ferreira, 2010], [Banet e Ayuso, 2000].

Consequentemente, o aprendizado da Genética Clássica (subárea da Biologia), torna-se um processo desafiador, em virtude do grande número de conceitos, dificultando a compreensão por parte dos alunos [Temp, 2011]. Diante disto, a área interdisciplinar das Tecnologias assistivas têm sido uma grande aliada nesse processo. Conceitualmente, é uma área interdisciplinar, com o objetivo de desenvolver recursos, metodologias, serviços, dentre outros, adequados à pessoa com deficiência [CORDE, 2009], como evidenciado nos estudos de [Nogueira e Nielsen, 2011], [Júnior et. al, 2018], [Araujo, et al., 2019; ] , [Roberto e Monteiro, 2019].

Como possíveis fontes de soluções que implementam tecnologias assistivas, o desenvolvimento de aplicações mobile apresenta-se como uma alternativa viável na superação de barreiras no ensino [Damaceno et. al, 2018] . A exemplo, algumas soluções relacionadas foram propostas para a Genética: Os autores Sánchez e Aguayo (2008), desenvolveram o AudioGene, um aplicativo móvel com foco no aprendizado dos conceitos básicos de genética. Em consonância, Correa e colaboradores (2015) um sistema de avaliação didática Acessível e Portátil, sobre conceitos de Genética, para pessoas com deficiência visual, utilizando como estudo de caso a plataforma Android. O trabalho de Alnfiaia e Sampalli (2017) por sua vez propõe a ferramenta BrailleEnter, um teclado acessível aos deficientes visuais, com foco na cegueira total.

Diante das abordagens apresentadas, destaca-se que a atual proposta, se difere das demais citadas, por ser uma ferramenta de auxílio para o ensino e aprendizagem de Genética Clássica a deficientes visuais, com cegueira total, podendo ser utilizada dentro ou fora do contexto de sala de aula. Ao professor é uma solução de apoio a outro método utilizado, ao aluno oferece a possibilidade de rever conceitos e exercitar os conhecimentos relacionados a disciplina em questão.

\section{A Genética Clássica e as Leis de Mendel}

$\mathrm{Na}$ genética clássica são abordados os princípios dos estudos que decorre dos experimentos, descobertas e teorias formuladas por Gregor Mendel em 1822, que tratam dos conceitos da transmissão de características de forma hereditária. Através de observações e experimentos, Mendel provou que a herança tem como base fatores específicos que são independentes uns dos outros, conclusão essa obtida através do estudo com a planta Pisum sativum, popularmente chamada de ervilha [Allen, 2003].

Os estudos de Mendel, com a planta ervilha, provou que a herança tem como base fatores específicos que são independentes uns dos outros. Esta análise resultou no primeiro postulado: O Princípio da Segregação dos Caracteres, onde cada característica de um indivíduo é determinada por um par de fatores, sendo um herdado do progenitor materno e outro do progenitor paterno, ambos separados durante a formação dos gametas, possuindo a mesma probabilidade de ocorrência [Snustad e Simmons, 2008] , [Fridman, 2012]. 
Com o segundo postulado (Segregação Independente), Mendel descobre que durante a formação dos gametas, os pares das características se separam independentes uns dos outros, recebendo cada gameta uma característica de cada par, sendo que os possíveis tipos de gametas serão formados nas mesmas proporções [Snustad e Simmons, 2008], [Fridman, 2012].

No âmbito educacional, o ensino dos princípios de Mendel são abordados na disciplina de Genética, integrando o componente curricular do Ensino Médio. E a forma de abordagem ocorre através de cruzamentos, onde é possível prever os resultados entre linhagens de diferentes organismos, isso acontece fazendo uso dos métodos da Probabilidade, Quadro de punnett e Linha bifurcada [Snustad e Simmons, 2008].

O quadro de punnet permite representar os gametas e combiná-los de maneira sistemática, gerando combinações possíveis dos genótipos dos indivíduos envolvidos [Allen, 2003]. Este é o método utilizado como abordagem, para estudo das leis mendelianas proposto pela aplicação desenvolvida.

\section{Metodologia}

Nesta seção são apresentados os procedimentos metodológicos utilizados para desenvolvimento e validação da aplicação. Estes foram divididos nas etapas de fundamentação teórica, levantamento de requisitos, modelagem, desenvolvimento e validação.

\subsection{Descrição das etapas}

A partir da etapa de fundamentação teórica, a próxima etapa realizada foi o levantamento de requisitos, que ocorreu no Centro de Atendimento à Pessoas com Necessidades Educacionais Especiais (NAPNE) do Instituto Federal de Educação, Ciência e Tecnologia do Pará, utilizando a técnica de entrevista semiestruturada [Manzini, 2004], que incluiu a participação de uma professora da área de ciências biológicas e estudantes com cegueira total. A escolha da Instituição Federal citada como cenário para este trabalho, justifica-se pelo uso de tecnologias assistivas para o atendimento a estudantes com deficiência, bem como pela equipe de educação inclusiva, atuante na mesma.

Em seguida, fez-se uso da Linguagem de Modelagem Unificada (UML), para construção da etapa de modelagem. A etapa de desenvolvimento foi baseada no modelo interativo [Sommerville, 2013], por meio da IDE Android Studio (versão 3.4.1), com a Linguagem de programação Java. O sintetizador de fala utilizado foi o "Text to Speech" ("TTS") do Google, e a biblioteca de gestos nativa do Android. Junto com estas, alguns métodos foram implementados, como OnSwipeTopE, OnSwipeTopD, OnSwipeBottomE e LGesture.

Posteriormente, na etapa de validação, o instrumento de coleta de dados utilizado foi o questionário SUS (System Usability Scale), para coletar opiniões e preferências dos usuários com base em seus níveis de satisfação em relação às interações com a aplicação testada. O questionário é composto por dez afirmações relacionadas a aplicação avaliada, e o usuário responde de acordo com a escala de Likert, composta por cinco pontos: 1discordo plenamente, 2- discordo, 3- neutro, 4-concordo e 5- concordo plenamente [Likert, 1932], [Brooke, 1996]. 
O método de pontuação do SUS é baseado nas respostas dos usuários às afirmações, classificadas em tons positivos e negativos. Para as afirmações positivas (1, $3,5,7,9)$, o valor de um é subtraído da resposta do usuário (R-1), e para afirmações negativas $(2,4,6,8,10)$, a resposta do usuário é subtraída de cinco (5-R). Em seguida, os valores que correspondem às dez afirmações são somados, e o total multiplicado por 2,5. O resultado geral representa a pontuação final do SUS, que varia em uma escala de 0 a 100 pontos [Sauro \& Lewis, 2012].

\section{BioBlu: Biology Teaching App to Aid Blind Users}

A interface da aplicação foi construída de forma a ser um modelo alternativo, complementar ao método de ensino do conteúdo de Genética Clássica para deficientes visuais. Durante processo de levantamento de requisitos a professora ressaltou que em virtude de não ser possível a representação em braille de alguns desenhos específicos necessários para explanar sobre alguns assuntos relacionados a Genética, uma alternativa é a confecção de modelos táteis para uso em sala de aula. Esses modelos são criados pelos próprios alunos com auxílio do NAPNE (Núcleo de Acessibilidade), realizando a impressão em Braille de algumas informações que compõe uma legenda.

A Figura 1 exibe o mapa dos cruzamentos mendelianos, um dos modelos confeccionados para uso em uma de suas aulas. Para desenvolver os modelos, a professora ressalta que sempre faz uso de materiais de baixo custo e de texturas variadas, para facilitar a assimilação pelo aluno. Para estes modelos ilustrados abaixo, foram utilizados diversos materiais, dentre eles, isopor, tampinha de refrigerante de tamanhos variados e velcro.

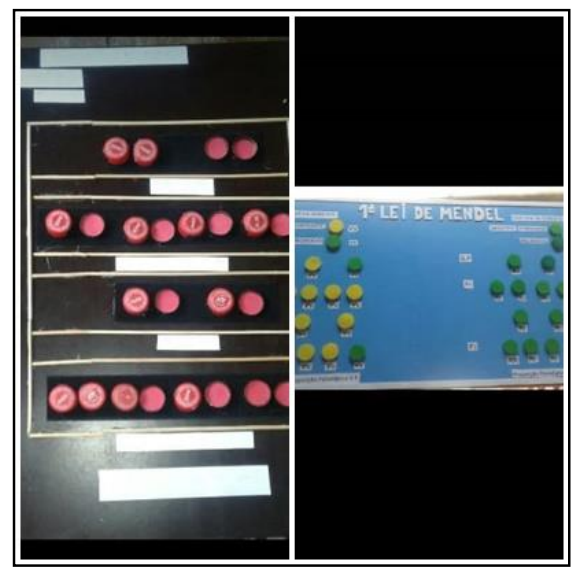

Figura 1. Modelos Leis de Mendel

Uma das dificuldades relatada pela professora, é que apesar da enorme contribuição e auxílio proporcionado pelos modelos táteis para compreensão dos conteúdos, sempre existe a necessidade de confecção de novos modelos, de acordo com o assunto a ser ministrado, geralmente utiliza-se somente um modelo por aula. Ressaltase ainda a dificuldade de manuseio dos modelos durante as aulas, em virtude de suas dimensões.

Em consequência, a interface da aplicação, foi desenvolvida de forma a fornecer os recursos necessários para apoiar o processo de ensino e aprendizagem, da Primeira e 
Segunda Lei de Mendel. Sendo possível a resolução de questões, tutoriais de aprendizagem e revisão dos principais conceitos da genética clássica.

O menu principal da aplicação (Figura 2-a) é constituída pela tela de ambientação, que apresenta os mecanismos de interação da aplicação, de forma que o usuário possa compreender os gestos necessários para utilização da mesma. A opção de Primeira Lei é composta pela opção de treino, cruzamento, e resolução de questões. De forma similar a interface de Segunda Lei de Mendel possui as mesmas funcionalidades, adequadas ao segundo postulado de Mendel. O conteúdo sobre alelos múltiplos, é apresentado na opção "Polialelia". Neste caso, a aplicação disponibiliza o problema da pelagem em coelhos para abordar o assunto (Figura 2-b).

A opção de configuração permite ao usuário, escolher o nível de velocidade de reprodução do feedback sonoro (iniciante, intermediário ou avançado), de acordo com sua experiência na utilização de aplicativos com leitor de tela. Esta tela é exibida automaticamente quando o usuário executa a aplicação pela primeira vez. Por fim, alguns conceitos importantes sobre o estudo da genética clássica, são apresentados na opção "revisão de conceitos".

O BioBlu faz uso de gestos e feedback sonoro para possibilitar ao aluno, realizar cruzamentos livres ou resolver questões. A opção de cruzamento está presente nas Leis Mendelianas e no módulo de Polialelia. Este ambiente permite que seja informada uma questão aleatória sobre as leis mendelianas. A exemplo o professor poderá informar verbalmente uma questão para o aluno, que fará uso desta área para resolução (Figura 2c).

Ao iniciar a resolução de uma questão neste modo, o usuário precisará definir uma letra que representará um alelo, posteriormente a aplicação exibirá uma área livre para que ele possa resolver a questão proposta. Para selecionar alelos do tipo dominante, o usuário realizará o gesto de "deslizar o dedo para cima ou para baixo", do lado esquerdo da tela. De forma similar, o mesmo gesto executado no lado direito da tela, possibilita a seleção de alelos do tipo recessivo. Estes e demais mecanismos de interação, são detalhados na opção de ambientação da aplicação.

A app disponibiliza a resolução de questões pré-estabelecidas na aplicação, através da opção "questões" (Figura 2-d), opção comum na interface de primeira e segunda lei. Para cada ação realizada, o usuário recebe feedback sonoro solicitando a confirmação do par de alelos selecionado, e ao final, a aplicação informa sobre o erro ou acerto da resolução. Mais detalhes sobre os mecanismos e forma de interação, estão detalhados no manual da aplicação, disponível em: https://www.lds.ndae.ufpa.br/tools.

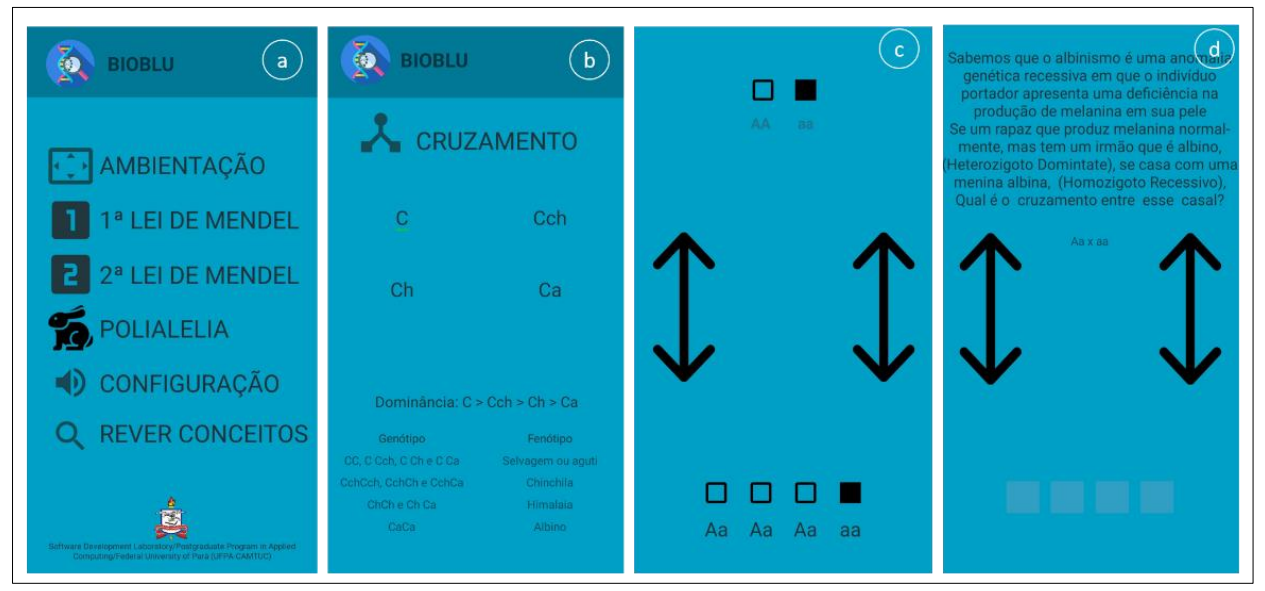

Figura 2. Telas da aplicação BioBlu 


\section{Avaliação da aplicação}

Após finalizado o desenvolvimento, o BioBlu foi submetido a testes de usabilidade, seguindo métricas estabelecidas por Nielsen (1990), os mesmos foram realizados na Associação de Deficientes Visuais (ADVASP), em Tucuruí, Pará. A aplicação foi utilizada por cinco participantes com deficiência visual (cegueira total) em faixas etárias variadas, recrutados com apoio e assistência das instituições (IFPA/Tucuruí e ADVASP). Do total, somente um participante não apresentava experiência no uso frequente de smartphones. Todos estavam de alguma forma inseridos em contexto escolar, sendo estudante do ensino médio, alguns cursando graduação, outros já com formação superior na área de Biologia.

Os testes seguiram um protocolo, onde inicialmente, a aplicação era apresentada, aos participantes envolvidos, e os mesmos tinham um tempo livre para exploração e familiaridade com a ferramenta. Posteriormente, foram propostas a execução das seguintes tarefas: i) configurar a velocidade de fala da aplicação, ii) realizar o processo de ambientação, iii) acessar a opção "rever conceitos" para relembrar os conceitos básicos relacionados ao assunto, iv) Resolver duas questões, referente à primeira e segunda lei, respectivamente.

Ao término das tarefas, o questionário SUS foi aplicado para medir a usabilidade do aplicativo. A escolha pela utilização dessa técnica justifica-se pela mesma ser composta de apenas dez perguntas, o que torna sua aplicação rápida, além de ser amplamente utilizada por vários profissionais no campo da interação homem-computador (IHC) [Finstad, 2006], [Bangor et. al, 2008].

Em relação a pontuação individual de cada participante, é possível inferir que o índice de aceitação da aplicação foi satisfatório, conforme observado na figura 3 . Ao considerarmos a média aritmética dos cinco participantes resultante do uso da aplicação, obtém-se o valor 79.0, evidenciando um nível de usabilidade adequada. Essa classificação é baseada no resultado obtido com as pontuações finais do questionário SUS. Segundo Bangor (2008), se a pontuação final obtida estiver entre 80 e 90 pontos, a aplicação tem a melhor usabilidade possível, enquanto pontuações entre 70 e 80 evidenciam uma usabilidade adequada, podendo precisar de algumas melhorias. Já para pontuações abaixo de 60 mostram um grau não aceitável de usabilidade [Bangor, 2008].

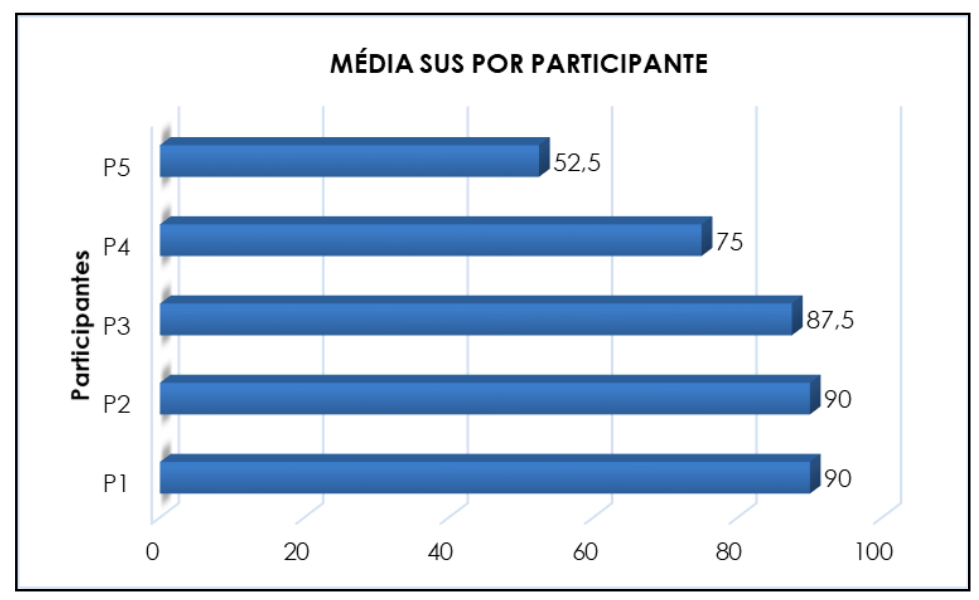

Figura 3. Média SUS por participante 
É possível ainda, relacionarmos cada afirmação contida no questionário SUS, com as heurísticas propostas por Nielsen e Molich (1990), tais que: facilidade de aprendizado (afirmações 3, 4, 7 e 10), eficiência (afirmações 5, 6 e 8), facilidade de memorização (afirmação 2), minimização de erros (afirmação 6) e satisfação (afirmações 1, 4 e 9) [Tenório et. al, 2010].

Os resultados desta correlação entre as afirmações levantadas com a aplicação do questionário SUS e as heurísticas propostas por Nielsen e Molich (1990), assim como os resultados obtidos na avaliação inicial da interface, e documentação de requisitos estão disponíveis de forma detalhada em:

https://drive.google.com/drive/folders/1Tr-FKglljepVLP2G9ZOvbK55VdOFK114?usp=sharing

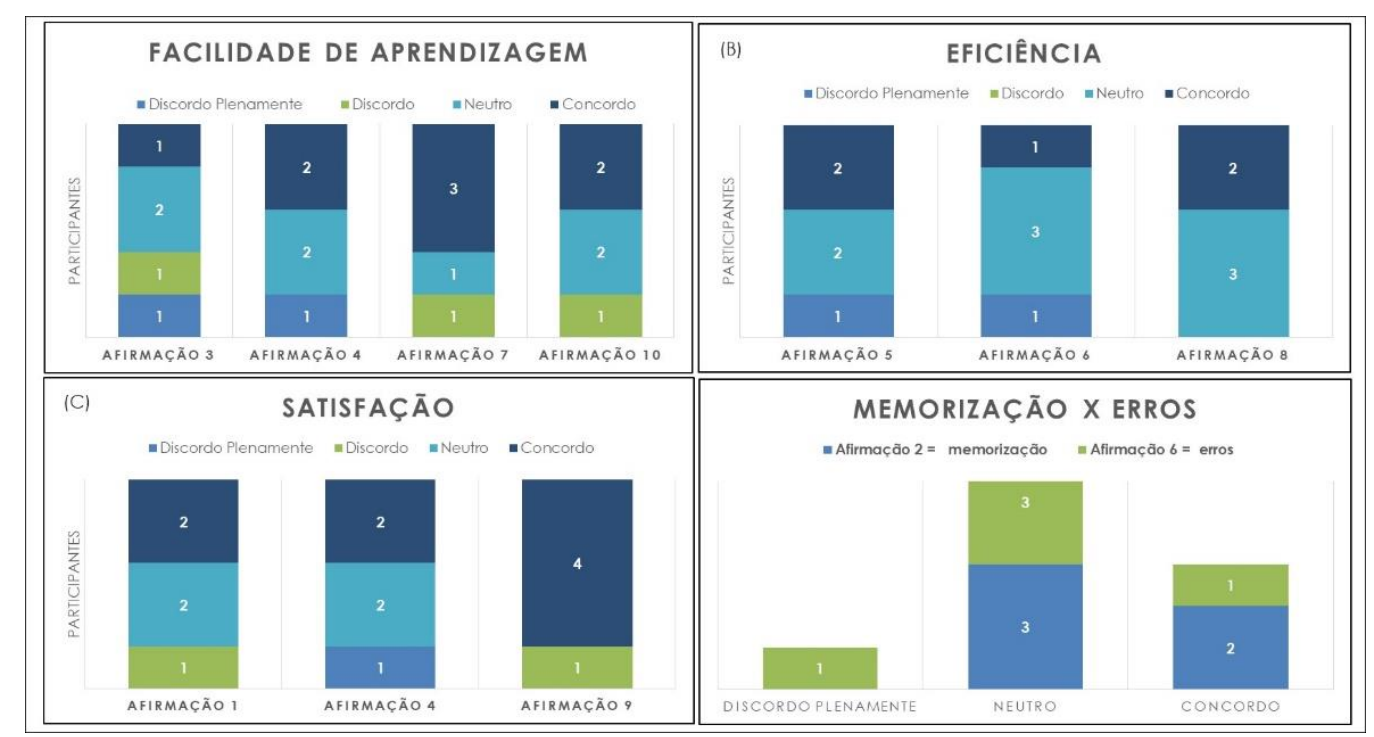

Figura 4. Escalas de notas por participantes

A partir da análise das respostas dos participantes relacionadas com a heurística: facilidade de aprendizagem (Figura 4 -A), obteve-se que: Para a afirmação três ("achei o aplicativo fácil de usar"), o índice de concordância foi de $20 \%$, sendo que dois dos cinco participantes (40\%) optaram pela neutralidade. Na afirmação quatro ("Preciso de ajuda técnica de alguém para usar o aplicativo"), dois participantes (40\%) concordaram com isso, enquanto outros dois optaram pela neutralidade, relatando que o recurso de "ajuda" presente na aplicação forneceu os esclarecimentos necessários, não necessitando portanto de auxílio extra.

Para a afirmação sete ("É possível aprender rapidamente como usar essa aplicação"), a taxa de concordância foi de $60 \%$. Três participantes relataram que e foi possível aprender a usar a aplicação sem dificuldades, após um período de exploração e quando necessário, recorriam as informações contidas na opção de "ambientação" da aplicação.

Quanto à afirmação dez ("Preciso aprender muitas coisas antes de usar a aplicação"), dois participantes concordaram (40\%), o que evidencia, a necessidade de conhecimento prévio sobre o assunto da disciplina ministrada em sala de aula pelo docente. Dois participantes optaram pela neutralidade, pois acharam importante 
relembrar o uso do método de resolução do quadro de punnet, evitando assim frustrações ao tentar resolver as questões propostas sem o conhecimento prévio associado.

Considerando a heurística relacionada à eficiência (Figura 4 - B): Para a afirmação cinco ("Conseguir utilizar bem as funções que a aplicação apresenta"), $40 \%$ dos participantes concordaram, pois não tiveram problemas quanto ao uso da aplicação. Por sua vez, dois participantes preferiram manter-se neutros, haja vista que para a utilização de algumas funções precisaram recorrer as formas de "ajuda" presentes na aplicação.

Para a afirmação seis ("A aplicação apresenta muitas falhas"), $20 \%$ dos participantes concordaram, e 60\% escolheram a opção "neutra", uma vez que conseguiram utilizar a aplicação sem problemas como: travamentos, erros no retorno auditivo, etc., no entanto em algum momento durante o uso, sentiram que mais recursos poderiam existir com o intuito de tornar a aplicação mais sucinta, como por exemplo a simplificação das orientações, já implantadas na versão final da App, presentes na interface de tutorial. Na afirmação oito ("Eu acho que a aplicação é difícil de ser utilizada") $40 \%$, dos participantes concordam com a afirmação, ressaltando algumas dificuldades, ocasionados pelo não domínio total no uso de smartphones e pela necessidade de conhecimento quanto ao assunto.

Para a heurística de satisfação (Figura 4-C), quanto à afirmação ("Gostaria de usar esse aplicativo com frequência"), $40 \%$ dos participantes concordaram com a afirmação. Outros 40\% escolheram a opção "neutra", pois enfatizaram que atualmente não há necessidade do uso frequente desse tipo de aplicação. No entanto, se no momento da aplicação dessa metodologia eles estivessem inseridos no contexto do estudo da área de abordagem, a aplicação seria essencial para auxiliar suas atividades.

A afirmação nove ("Fiquei satisfeito com a aplicação") obteve $80 \%$ de satisfação dos participantes. Em relação à facilidade de memorização (afirmação 2) e minimização de erros (afirmação 6), as taxas de concordância para ambas foram de $40 \%$. Os resultados obtidos com a análise apontam um índice positivo da aplicação BioBlu, como ferramenta de auxílio ao estudo da disciplina de Genética pelas pessoas com deficiência visual.

\section{Considerações Finais}

Este estudo apresenta uma tecnologia assistiva e seu uso no campo educacional, através do desenvolvimento de uma aplicação mobile, que auxilia o processo de ensino e aprendizagem da disciplina de genética clássica para pessoas com deficiência visual.

A interface do BioBlu, mostrou-se acessível quanto a abordagem dos assunto das Leis Mendelianas, bem como contribuiu para o aprendizado de alunos com deficiência visual, sendo possível sua utilização tanto em sala de aula para apoiar a didática do educador, como também no ambiente doméstico, onde o aluno pode revisitar conceitos e estudar, resolvendo problemas e praticando o que aprendeu em sala de aula.

Aos professores da disciplina, o BioBlu pode ser utilizado de forma complementar ao método de ensino habitual, possibilitando ao educador definir qual a melhor maneira de uso da ferramenta em auxílio a sua prática pedagógica. Ressalta-se ainda que apesar da aplicação ser direcionada à pessoas com deficiência visual, a mesma pode utilizada também por videntes, contribuindo assim para consolidação de atividades colaborativas em sala de aula.

Em relação a trabalhos futuros, propõe-se uma investigação ampla, envolvendo o discente com cegueira total e o docente da disciplina de Genética, analisando a dinâmica 
em sala de aula de ambos quanto a utilização da ferramenta desenvolvida neste estudo, e validando aspectos da melhoria no aprendizado por parte do aluno com deficiência visual. Há ainda a possibilidade de expansão da aplicação, mediante a inclusão de novos módulos referentes a outros assuntos da Genética, de forma a consolidá-la como uma proposta colaborativa, bem como o desenvolvimento em curso de uma plataforma web para dinamizar a interação entre professor e aluno, enriquecendo ainda mais o conteúdo disponível na aplicação.

\section{References}

Allen, G. E. (2003). Mendel and modern genetics: The legacy for today. Endeavour, 27(2), 63-68. https://doi.org/10.1016/S0160-9327(03)00065-6

Araujo, P., Viana, W., Veras, N., Farias, E. J., \& Castro Filho, J. A. de. (2019). Exploring Students Perceptions and Performance in Flipped Classroom Designed with Adaptive Learning Techniques: A Study in Distributed Systems Courses. VIII Congresso Brasileiro de Informática Na Educação, (Cbie), 219. https://doi.org/10.5753/cbie.sbie.2019.219

Banet, E., \& Ayuso, E. (2000). Teaching genetics at secondary school: A strategy for teaching about the location of inheritance information. Science Education, 84(3), 313351.https://doi.org/10.1002/(SICI)1098-237X(200005)84:3<313::AIDSCE2>3.0.CO;2-N

Bangor, A., Kortum, P. T., \& Miller, J. T. (2008). An empirical evaluation of the system usability scale. International Journal of Human-Computer Interaction, 24(6), 574-594. https://doi.org/10.1080/10447310802205776

Brooke, J. (1996). SUS - A quick and dirty usability scale. Usability Evaluation in Industry, 189-194.

Cardinali, S. M. M.; Ferreira, A. C. (2010). A aprendizagem da célula pelos estudantes cegos utilizando modelos tridimensionais: um desafio .Revista Benjamin Constant. Ética., Rio de Janeiro, v. 46, n. 46, p. 5-12.

Carvalho, R. E. (2005). Educação Inclusiva com os Pingos nos Is.

CORDE. (2009). Tecnologia Assistiva (pp. 1-138). pp. 1-138.

Damaceno, R. J. P., Braga, J. C., \& Mena-Chalco, J. P. (2018). Mobile device accessibility for the visually impaired: problems mapping and recommendations. Universal Access in the Information Society, 17(2), 421-435. https://doi.org/10.1007/s10209-017-0540-1

Fávero, E. A., Pantoja, L., \& Mantoan, M. T. E. (2007). Atendimento Educacional Especializado: Aspectos Legais e orientaçao pedagógica. São Paulo: MEC/SEESP.

Finstad, K. (2006). The system usability scale and non-native English speakers. Journal of Usability Studies, 1(4), 185-188.

Fridman, C. (2012). As 1a E 2a Leis de Mendel e Conceitos básicos de Citogenética. Evolução Das Ciências II, 7-8.

Júnior, S. S., Sérgio, C. C., Pinto, S., \& Braz, R. M. M. (2018). A Computação como Tecnologia Assistiva na construção de um Mapa Tátil para os Alunos com Deficiência Visual nos Estudos de um Mapa Político da Região Sudeste do Brasil. VI Congresso 
IX Congresso Brasileiro de Informática na Educação (CBIE 2020)

Anais do XXXI Simpósio Brasileiro de Informática na Educação (SBIE 2020)

Brasileiro de Informática Na Educação, (Cbie), 294-301. https://doi.org/10.5753/cbie.wcbie.2018.294

Laplane, A. L., \& Batista, C. G. (2008). Ver, não ver e aprender: a participação de crianças com baixa visão e cegueira na escola. 28, 209-227. Retrieved from http://www.cedes.unicamp.br

Likert, R. (1932). A Techinique for the measurement of attitudes.

Manzini, E. J. (2004). Entrevista semi-estruturada: análise de objetivos e de roteiros. 2, 10.

Mendonça, A., Miguel, C., Neves, G., Micaelo, M., \& Reino, V. (2008). Alunos cegos e com baixa visão - Orientações curriculares (Direcção-Geral de Inovação e de Desenvolvimento Curricular \& Direcção de Serviços da Educação Especial e do Apoio Sócio-Educativo, Eds.). Retrieved from http://www.deficienciavisual.pt/x-txt-abaOrientacoesCurricularesCegosBxV.pdf

Nielsen J, Molich R. (1990). Heuristic Evaluation of User Interfaces. Cross-Cultural Human-Computer Interact User Exp Des.163-6. https://doi.org/10.1201/b18059-15.

Nogueira, A. S., \& Nielsen, R. D. J. (2011). Homero Software : Auxiliando o Ensino de portadores de cegueira. Anais Do XXII SBIE-XVII WIE, 1612-1615.

Roberto, J., \& Monteiro, A. M. (2019). Requisitos para o Desenvolvimento de Objetos de Aprendizagem Reutilizáveis para Dispositivos Móveis. VIII Congresso Brasileiro de Informática Na Educação, (Cbie), 81-88. https://doi.org/10.5753/cbie.sbie.2019.81

Sauro, J., \& Lewis, J. R. (2012). Quantifying the user experience (Elsevier, Ed.).

Snustad, D. P., \& Simmons, M. J. (2008). Fundamentos de Genética (7th ed.; G. Koogan, Ed.). Rio de Janeiro.

Sommerville, I. (2013). Engenharia de software (9a; Pearson, Ed.). São Paulo.

Temp, D. S. (2011). Facilitando a aprendizagem de Genética: uso de um modelo didático e análise dos recursos presentes em livros de Biologia. Dissertação (Mestrado em Educação em Ciências) - Universidade Federal de Santa Maria, Santa Maria, RS.

Tenório, J. M., Cohrs, F. M., Sdepanian, V. L., Pisa, I. T., \& Marin, H. D. F. (2010). Desenvolvimento e Avaliação de um Protocolo Eletrônico para Atendimento e Monitoramento do Paciente com Doença Celíaca. Revista de Informática Teórica e Aplicada, 17(2), 210. https://doi.org/10.22456/2175-2745.12119

WHO. (2019). World report on vision. Retrieved from https://www.who.int/publicationsdetail/world-report-on-vision 